\title{
A Parameterized View on Matroid Optimization Problems
}

\author{
Dániel Marx \\ Institut für Informatik, \\ Humboldt-Universität zu Berlin, \\ Unter den Linden 6, 10099 \\ Berlin, Germany. \\ dmarx@informatik.hu-berlin.de
}

\begin{abstract}
Matroid theory gives us powerful techniques for understanding combinatorial optimization problems and for designing polynomialtime algorithms. However, several natural matroid problems, such as 3-matroid intersection, are NP-hard. Here we investigate these problems from the parameterized complexity point of view: instead of the trivial $O\left(n^{k}\right)$ time brute force algorithm for finding a $k$-element solution, we try to give algorithms with uniformly polynomial (i.e., $f(k) \cdot n^{O(1)}$ ) running time. The main result is that if the ground set of a represented matroid is partitioned into blocks of size $\ell$, then we can determine in $f(k, \ell) \cdot n^{O(1)}$ randomized time whether there is an independent set that is the union of $k$ blocks. As consequence, algorithms with similar running time are obtained for other problems such as finding a $k$-set in the intersection of $\ell$ matroids, or finding $k$ terminals in a network such that each of them can be connected simultaneously to the source by $\ell$ disjoint paths.
\end{abstract}

\section{Introduction}

Many of the classical combinatorial optimization problems can be studied in the framework of matroid theory. The polynomial-time solvability of finding minimum weight spanning trees, finding perfect matchings, and certain connectivity problems all follow from the general algorithmic results on matroids.

Deciding whether there is an independent set of size $k$ in the intersection of two matroids can be done in polynomial time, but the problem becomes NPhard if we have to find a $k$-element set in the intersection of three matroids. Of course, the problem can be solved in $n^{O(k)}$ time by brute force, hence it is polynomial-time solvable for every fixed value of $k$. However, the running time is prohibitively large, even for small values of $k$ (e.g., $k=10$ ) and moderate values of $n$ (e.g., $n=1000$ ). The aim of parameterized complexity is to identify problems that can be solved in uniformly polynomial time for every fixed value of the problem parameter $k$, that is, the running time is of the form $f(k) \cdot n^{O(1)}$. A problem that can be solved in such time is called fixed-parameter tractable. Notice the huge qualitative difference between running times such as $O\left(2^{k} \cdot n^{2}\right)$ 
and $n^{k}$ : the former can be efficient even for, say, $k=15$, while the latter has no chance of working. For more background on parameterized complexity, see [1].

The question that we investigate in this paper is whether the NP-hard matroid optimization problems can be solved in uniformly polynomial time, if the parameter is the size of the object that we are looking for. The most general result is the following:

Theorem 1 (Main). Let $M(E, \mathcal{I})$ be a matroid where the ground set is partitioned into blocks of size $\ell$. Given a representation $A$ of $M$, it can be determined in $f(k, \ell) \cdot\|A\|^{O(1)}$ randomized time whether there is an independent set that is the union of $k$ blocks. ( $\|A\|$ denotes the length of $A$ in the input.)

For $\ell=2$, this problem is exactly the matroid parity problem, which is polynomialtime solvable for represented matroids [4]. For $\ell \geq 3$, the problem is NP-hard.

As applications of the main result, we show that the following problems are also solvable in $f(k, \ell) \cdot n^{O(1)}$ randomized time:

1. Given a family of subsets each of size at most $\ell$, find $k$ of them that are pairwise disjoint.

2. Given a graph $G$, find $k$ (edge) disjoint triangles in $G$.

3. Given $\ell$ matroids over the same ground set, find a set of size $k$ that is independent in each matroid.

4. Feedback Edge Set with Budget Vectors: given a graph with $\ell$ dimensional cost vectors on the edges, find a feedback edge set of size at most $k$ such that the total cost does not exceed a given vector $C$ (see Section 5.3 for the precise definition).

5. Reliable Terminals: select $k$ terminals and connect each of them to the source with $\ell$ paths such that these $k \cdot \ell$ paths are pairwise disjoint.

The fixed-parameter tractability of the first two problems is well-known: they can be solved either with color coding or using representative systems. However, it is interesting to see that randomized fixed-parameter tractability can be obtained as a straightforward corollary of our results on matroids. We are not aware of any parameterized investigations of the last three problems.

The algorithm behind the main result is inspired by the technique of representative systems introduced by Monien [6] (see also [8,5] and [1, Section 8.2]). Iteratively for $i=1,2, \ldots, \ell$, we construct a collection $\mathcal{S}_{i}$ that contains independent sets arising as the union of $i$ blocks (if there are such independent sets). The crucial observation is that we can ensure that the size of each $\mathcal{S}_{i}$ is at most a constant depending only on $k$ and $\ell$. In [5], this bound is obtained using Bollobás' Inequality. In our case, the bound can be obtained using a linear-algebraic generalization of Bollobás' Inequality due to Lovász [3, Theorem 4.8] (see also [2, Chapter 31, Lemma 3.2]). However, we need an algorithmic way of bounding the size of the $\mathcal{S}_{i}$ 's, hence we do not state and use these inequalities here, but rather reproduce the proof of Lovász in an algorithmic form (Lemma 12). The proof of this lemma is a simple application of multilinear algebra.

The algorithms that we obtain are randomized in the sense that they use random numbers and there is a small probability of not finding a solution even if it 
exists. The randomized nature of the algorithm comes from the fact that we rely on the Zippel-Schwartz Lemma in some of the operations involving matroid representations. Additionally, when working with representations over finite fields, then some of the algebraic operations are most conveniently done randomized. As the main result is randomized, we do not discuss whether these miscellaneous algebraic operations can be derandomized.

Section 2 summarizes the most important notions of matroid theory. Section 3 discusses how certain operations can be performed on the representations of matroids. Most of these constructions are either easy or folklore. The reason why we discuss them in detail is that we need these results in algorithmic form. The main result is presented in Section 4. In Section 5, the randomized fixedparameter tractability of certain problems are deduced as corollaries.

\section{Preliminaries}

A matroid $M(E, \mathcal{I})$ is defined by a ground set $E$ and a collection $\mathcal{I} \subseteq 2^{E}$ of independent sets satisfying the following three properties:

(I1) $\emptyset \in \mathcal{I}$

(I2) If $X \subseteq Y$ and $Y \in \mathcal{I}$, then $X \in \mathcal{I}$.

(I3) If $X, Y \in \mathcal{I}$ and $|X|<|Y|$, then $\exists e \in Y \backslash X$ such that $X \cup\{e\} \in \mathcal{I}$.

An inclusionwise maximal set of $\mathcal{I}$ is called a basis of the matroid. It can be shown that the bases of a matroid all have the same size. This size is called the rank of the matroid $M$, and is denoted by $r(M)$. The $\operatorname{rank} r(S)$ of a subset $S$ is the size of the largest independent set in $S$.

The definition of matroids was motivated by two classical examples. Let $G(V, E)$ be a graph, and let a subset $X \subseteq E$ of edges be independent if $X$ does not contain any cycles. This results in a matroid, which is called the cycle matroid of $G$. The second example comes from linear algebra. Let $A$ be a matrix over an arbitrary field $F$. Let $E$ be the set of columns of $A$, and let $X \subseteq E$ be independent if these columns are linearly independent. The matroids that can be defined by such a construction are called linear matroids, and if a matroid can be defined by a matrix $A$ over a field $F$, then we say that the matroid is representable over $F$. In this paper we consider only representable matroids, hence matroids are given by a matrix $A$ over a field $F$. To avoid complications involving the representations of the elements in the matrix, we assume that $F$ is either a finite field or the rationals. We denote by $\|A\|$ the size of the representation $A$ : the total number of bits required to describe all elements of the matrix.

We say that an algorithm is randomized polynomial time if the running time can be bounded by a polynomial of the input size and the error parameter $P$, and it produces incorrect answer with probability at most $2^{-P}$. Most of the randomized algorithms in this paper are based on the following lemma:

Lemma 2 (Zippel-Schwartz [12,10]). Let $p\left(x_{1}, \ldots, x_{n}\right)$ be a nonzero polynomial of degree $d$ over some field $F$, and let $S$ be an $N$ element subset of $F$. If each $x_{i}$ is independently assigned a value from $S$ with uniform probability, then $p\left(x_{1}, \ldots, x_{n}\right)=0$ with probability at most $d / N$. 


\section{Representation Issues}

The algorithm in Section 4 is based on algebraic manipulations, hence it requires that the matroid is given by a linear representation in the input. Therefore, in the proof of the main result and in its applications, we need algorithmic results on how to find representations for certain matroids, and if some operation is performed on a matroid, then how to obtain a representation of the result.

\subsection{Dimension}

The rank of a matroid represented by an $m \times n$ matrix is a most $m$ : if the columns are $m$-dimensional vectors, then more than $m$ of them cannot be independent. Conversely, every linear matroid of rank $r$ has a representation with $r$ rows:

Proposition 3. Given a matroid $M$ of rank $r$ with a representation $A$ over $F$, we can find in polynomial time a representation $A^{\prime}$ over $F$ having $r$ rows.

\subsection{Increasing the Size of the Field}

The applications of Lemma 2 requires $N$ to be large, so the probability of accidentally finding a root is small. However, $N$ can be large only if the field $F$ contains a sufficient number of elements. Therefore, if a matroid representation is given over some small field $F$, then we need a method of transforming this representation to a representation over a field $F^{\prime}$ having at least $N$ elements.

Let $|F|=q$ and let $n=\left\lceil\log _{q} N\right\rceil$. We construct a field $F^{\prime}$ having $q^{n} \geq N$ elements. In order to do this, an irreducible polynomial $p(x)$ of degree $n$ over $F$ is required. Such a polynomial $p(x)$ can be found for example by the randomized algorithm of Shoup [11] in time polynomial in $n$ and $\log q$. Now the ring of degree $n$ polynomials over $F$ modulo $p(x)$ is a field $F^{\prime}$ of size $q^{n}$. If a representation over $F$ is given, then each element can be replaced by the corresponding degree 0 polynomial from $F^{\prime}$, which yields a representation over $F^{\prime}$.

Proposition 4. Let $A$ be the representation of a matroid $M$ over some field $F$. For every $N$, it is possible to construct a representation $A^{\prime}$ of $M$ over some field $F^{\prime}$ with $\left|F^{\prime}\right| \geq N$ in $(\|A\| \cdot \log N)^{O(1)}$ randomized time.

\subsection{Direct Sum}

Let $M_{1}\left(E_{1}, \mathcal{I}_{1}\right)$ and $M_{2}\left(E_{2}, \mathcal{I}_{2}\right)$ be two matroids with $E_{1} \cap E_{2}=\emptyset$. The direct sum $M_{1} \oplus M_{2}$ is a matroid over $E:=E_{1} \cup E_{2}$ such that $X \subseteq E$ is independent if and only if $X \cap E_{1} \in \mathcal{I}_{1}$ and $X \cap E_{2} \in \mathcal{I}_{2}$. The notion can be generalized for the sum of more than two matroids.

Proposition 5. Given representations of matroids $M_{1}, \ldots, M_{k}$ over the same field $F$, a representation of their direct sum can be found in polynomial time.

\subsection{Uniform and Partition Matroids}

The uniform matroid $U_{n, k}$ has an $n$-element ground set $E$, and a set $X \subseteq E$ is independent if and only if $|X| \leq k$. Every uniform matroid is linear and can 
be represented over the rationals by a $k \times n$ matrix where the element in the $i$-th column of $j$-th row is $i^{(j-1)}$. Clearly, no set of size larger than $k$ can be independent in this representation, and every set of $k$ columns is independent, as they form a Vandermonde matrix.

A partition matroid is given by a ground set $E$ partitioned into $k$ blocks $E_{1}$, $\ldots, E_{k}$, and by $k$ integers $a_{1}, \ldots, a_{k}$. A set $X \subseteq E$ is independent if and only if $\left|X \cap E_{i}\right| \leq a_{i}$ holds for every $i=1, \ldots, k$. As this partition matroid is the direct sum of uniform matroids $U_{\left|E_{1}\right|, a_{1}}, \ldots, U_{\left|E_{k}\right|, a_{k}}$, we have

Proposition 6. A representation over the rationals of a partition matroid can be constructed in polynomial time.

\subsection{Dual}

The dual of a matroid $M(E, \mathcal{I})$ is a matroid $M^{*}\left(E, \mathcal{I}^{*}\right)$ over the same ground set where a set $B \subseteq E$ is a basis of $M^{*}$ if and only if $E \backslash B$ is a basis of $M$.

Proposition 7. Given a representation $A$ of a matroid $M$, a representation of the dual matroid $M^{*}$ can be found in polynomial time.

Proof. Let $r$ be the rank of the matroid $M$. By Prop. 3, it can be assumed that $A$ is of the form $\left(\begin{array}{ll}I_{r \times r} & B\end{array}\right)$, where $I_{r \times r}$ is the unit matrix of size $n \times n$, and $B$ is a matrix of size $r \times(n-r)$. Now the matrix $A^{*}=\left(B^{\top} I_{(n-r) \times(n-r)}\right)$ represents the dual matroid $M^{*}$, see any text on matroid theory (e.g., [9]).

\subsection{Truncation}

The $k$-truncation of a matroid $M(E, \mathcal{I})$ is a matroid $M^{\prime}\left(E, \mathcal{I}^{\prime}\right)$ such that $S \subseteq E$ is independent in $M^{\prime}$ if and only if $|S| \leq k$ and $S$ is independent in $M$.

Proposition 8. Given a matroid $M$ with a representation $A$ over a finite field $F$ and an integer $k$, a representation of the $k$-truncation $M^{\prime}$ can be found in randomized polynomial time.

Proof. By Prop. 3 and 4, it can be assumed that $A$ is of size $r \times n$ and the size of $F$ is at least $N:=2^{P} \cdot k n^{k}$. Let $R$ be a random matrix of size $k \times r$, where each element is taken from $F$ with uniform distribution. We claim that with high probability, $R A$ is a representation of the $k$-truncation. Since $R A$ cannot have more than $k$ independent columns, all we have to show is that a $k$-element set is independent in $M^{\prime}$ if and only if it is independent in $M$. Let $S$ be a set of size $k$, let $A_{0}$ be the $r \times k$ submatrix of $A$ formed by the corresponding $k$ columns, and let $B_{0}=R A_{0}$ be the corresponding $k$ columns in $R A$. If $S$ is not independent in $M$, then the columns of $B_{0}$ are not independent either. This means that $S$ is not independent in the matroid $M^{\prime}$ represented by $R A$. Assume now that $S$ is independent in $M$. The columns of $A_{0}$ are independent, thus $\operatorname{det} R A_{0} \neq 0$ with positive probability (e.g., there is a matrix $R$ such that $R A_{0}$ is the unit matrix). We use Lemma 2 to show that this probability is at least $1-2^{-P} / n^{k}$. The value 
$\operatorname{det} R A_{0}$ can be considered as a polynomial, with the $k r$ elements of the matrix $R$ being the variables. Since $\operatorname{det} R A_{0}$ is not always zero, the polynomial is not identically zero. As the degree of this polynomial is $k$, Lemma 2 ensures that $\operatorname{det} R A_{0}=0$ with probability at most $k / N=2^{-P} / n^{k}$. Thus the probability that a particular $k$-element independent set of $M$ is not independent in $M^{\prime}$ is at most $2^{-P} / n^{k}$. As $M$ has not more than $n^{k}$ independent set of size $k$, the probability that $M^{\prime}$ is not the $k$-truncation of $M$ is at most $2^{-P}$.

\subsection{Cycle Matroids}

The cycle matroid of $G(V, E)$ can be represented over the 2-element field: consider the $|V| \times|E|$ incidence matrix of $G$, where the $i$-th element of the $j$-row is 1 if and only if the $i$-th vertex is an endpoint of the $j$-th edge.

Proposition 9. Given a graph, a representation of the cycle matroid over the two element field can be constructed in polynomial time.

\subsection{Transversal Matroids}

Let $G(A, B ; E)$ be a bipartite graph. The transversal matroid $M$ of $G$ has $A$ as its ground set, and a subset $X \subseteq A$ is independent in $M$ if and only if there is a matching that covers $X$. That is, $X$ is independent if and only if there is an injective mapping $\phi: X \rightarrow B$ such that $\phi(v)$ is a neighbor of $v$ for every $v \in X$.

Proposition 10. Given a bipartite graph $G(A, B ; E)$, a representation of its transversal matroid can be constructed in randomized polynomial time.

Proof. Let $R$ be a $|B| \times|A|$ matrix, where the $i$-th element in the $j$-th row is

- a random integer between 1 and $N:=2^{P} \cdot|A| \cdot 2^{|A|}$ if the $i$-th element of $A$ and the $j$-th element of $B$ are adjacent, and

-0 otherwise.

We claim that with high probability, $R$ represents the transversal matroid of $M$. Assume that a subset $X$ of columns is independent. These columns have a $|X| \times|X|$ submatrix with nonzero determinant, hence there is at least one nonzero term in the expansion of this determinant. The nonzero term is a product of $|X|$ nonzero cells, and these cells define a matching covering $X$.

Assume now that $X \subseteq A$ is independent in the transversal matroid: it can be matched with elements $Y \subseteq B$. This means that the determinant of the $|Y| \times|X|$ submatrix $R_{0}$ of $R$ corresponding to $X$ and $Y$ has a term that is the product of nonzero elements. The determinant of $R_{0}$ can be considered as a polynomial of degree at most $|A|$, where the variables are the random elements of $R_{0}$. The existence of the matching and the corresponding nonzero term in the determinant shows that this polynomial is not identically zero. By Lemma 2 , the probability that the determinant of $R_{0}$ is zero is at most $2^{-P} / 2^{|A|}$, implying that the columns $X$ are independent with high probability. There are at most $2^{|A|}$ independent sets in $M$, thus the probability that not all of them are independent in the matroid represented by $R$ is at most $2^{-P}$. 


\section{The Main Result}

In this section we give a randomized fixed-parameter tractable algorithm for determining whether there are $k$ blocks whose union is independent, if a matroid is given with a partition of the ground set into blocks of size $\ell$. The idea is to construct for $i=1, \ldots, k$ the set $\mathcal{S}_{i}$ of all independent sets that arise as the union of $i$ blocks. A solution exists if and only if $\mathcal{S}_{k}$ is not empty. The set $\mathcal{S}_{i}$ is easy to construct if $\mathcal{S}_{i-1}$ is already known. The problem is that the size of $\mathcal{S}_{i}$ can be as large as $n^{\Omega(i)}$, hence we cannot handle sets of this size in uniformly polynomial time. The crucial idea is that we retain only a constant size subset of each $\mathcal{S}_{i}$ in such a way that we do not throw away any sets essential for the solution. The property that this reduced collection has to satisfy is the following:

Definition 11. Given a matroid $M(E, \mathcal{I})$ and a collection $\mathcal{S}$ of subsets of $E$, we say that a subsystem $\mathcal{S}^{*} \subseteq \mathcal{S}$ is $r$-representative for $\mathcal{S}$ if the following holds: for every set $Y \subseteq E$ of size at most $r$, if there is a set $X \in \mathcal{S}$ disjoint from $Y$ with $X \cup Y \in \mathcal{I}$, then there is a set $X^{*} \in S^{*}$ disjoint from $Y$ with $X^{*} \cup Y \in \mathcal{I}$.

That is, if an independent set in $\mathcal{S}$ can be extended to an independent set by $r$ new elements, then there is a set in $\mathcal{S}^{*}$ that can be extended by the same $r$ elements. 0-representative means that $\mathcal{S}^{*}$ is not empty if $\mathcal{S}$ is not empty. We use the following lemma to obtain a representative subcollection of constant size:

Lemma 12. Let $M$ be a linear matroid of rank $r+s$, and let $\mathcal{S}=\left\{S_{1}, \ldots, S_{m}\right\}$ be a collection of independent sets, each of size s. If $|\mathcal{S}|>\left(\begin{array}{c}r+s \\ s\end{array}\right)$, then there is a set $S \in \mathcal{S}$ such that $\mathcal{S} \backslash\{S\}$ is r-representative for $\mathcal{S}$. Furthermore, given a representation $A$ of $M$, we can find such a set $S$ in $f(r, s) \cdot(\|A\| m)^{O(1)}$ time.

Proof. Assume that $M$ is represented by an $(r+s) \times n$ matrix $A$ over some field $F$. Let $E$ be the ground set of the matroid $M$, and for each element $e \in E$, let $x_{e}$ be the corresponding $(r+s)$-dimensional column vector of $A$. Let $w_{i}=\bigwedge_{e \in S_{i}} x_{e}$, a vector in the exterior algebra of the linear space $F^{r+s}$. As every $w_{i}$ is the wedge product of $s$ vectors, the $w_{i}$ 's span a space of dimension at most $\left(\begin{array}{c}r+s \\ s\end{array}\right)$. Therefore, if $|\mathcal{S}|>\left(\begin{array}{c}r+s \\ s\end{array}\right)$, then the $w_{i}$ 's are not independent. Thus it can be assumed that some vector $w_{k}$ can be expressed as the linear combination of the other vectors.

We claim that if $S_{k}$ is removed from $\mathcal{S}$, then the resulting subsystem is $r$ representative for $\mathcal{S}$. Assume that, on the contrary, there is a set $Y$ of size at most $r$ such that $S_{k} \cap Y=\emptyset$ and $S_{k} \cup Y$ is independent, but this does not hold for any other $S_{i}$ with $i \neq k$. Let $y=\wedge_{e \in Y} x_{e}$. A crucial property of the wedge product is that the product of some vectors in $F^{r+s}$ is zero if and only if they are not independent. Therefore, $w_{k} \wedge y \neq 0$, but $w_{i} \wedge y=0$ for every $i \neq k$. However, $w_{k}$ is the linear combination of the other $w_{i}$ 's, thus, by the multilinearity of the wedge product, $w_{k} \wedge y \neq 0$ is a linear combination of the values $w_{i} \wedge y=0$ for $i \neq k$, which is a contradiction.

It is straightforward to make this proof algorithmic. First we determine the vectors $w_{i}$, then a vector $w_{k}$ that is spanned by the other vectors can be found by standard techniques of linear algebra. Let us fix a basis of $F^{r+s}$, and express 
the vectors $x_{e}$ as the linear combination of the basis vectors. The vector $w_{i}$ is the wedge product of $s$ vectors, hence, using the multilinearity of the wedge product, each $w_{i}$ can be expressed as the sum of $(r+s)^{s}$ terms. Each term is the wedge product of basis vectors of $F^{r+s}$; therefore, the antisymmetry property can be used to reduce each term to 0 or a basis vector of the exterior algebra. Thus we obtain each $w_{i}$ as a linear combination of basis vectors. Now Gaussian elimination can be used to determine the rank of the subspace spanned by the $w_{i}$ 's, and to check whether the rank remains the same if one of the vectors is removed. If so, then the set corresponding to this vector can be removed from $\mathcal{S}$, and the resulting subsystem $\mathcal{S}^{*}$ is representative for $\mathcal{S}$. The running time of the algorithm can be bounded by a polynomial of the number of vectors $n$, the number of terms in the expression of a $w_{i}\left(\right.$ i.e., $\left.(r+s)^{s}\right)$, the dimension of the subspace spanned by the $w_{i}$ 's (i.e., $\left(\begin{array}{c}r+s \\ s\end{array}\right)$ ), and the size of the representation of $M$. Therefore, the algorithm is polynomial-time for every fixed value of $r$ and $s$.

Now we are ready to prove the main result:

Proof (of Theorem 1). First we obtain a representation $A^{\prime}$ for the $k \ell$-truncation of the matroid. By Prop 8, this can be done in time polynomial in $\|A\|$. Using $A^{\prime}$ instead of $A$ does not change the answer to the problem, as we consider the independence of the union of at most $k$ blocks. However, when invoking Lemma 12, it will be important that the elements are represented as $k \ell$-dimensional vectors.

For $i=1, \ldots, k$, let $\mathcal{S}_{i}$ be the set system containing those independent sets that arise as the union of $i$ blocks. Clearly, the task is to determine whether $\mathcal{S}_{k}$ is empty or not. For each $i$, we construct a subsystem $\mathcal{S}_{i}^{*} \subseteq \mathcal{S}_{i}$ that is $(k-i) \ell$ representative for $\mathcal{S}_{i}$. As $\mathcal{S}_{k}^{*}$ is 0-representative for $\mathcal{S}_{k}$, the emptiness of $\mathcal{S}_{k}$ can be checked by checking whether $\mathcal{S}_{k}^{*}$ is empty.

The set system $\mathcal{S}_{1}$ is easy to construct, hence we can take $\mathcal{S}_{1}^{*}=\mathcal{S}_{1}$. Assume now that we have a set system $\mathcal{S}_{i}^{*}$ as above. The set system $\mathcal{S}_{i+1}^{*}$ can be constructed as follows. First, if $\left|\mathcal{S}_{i}^{*}\right|>\left(\begin{array}{c}i \ell+(k-i) \ell \\ i \ell\end{array}\right)=\left(\begin{array}{c}k \ell \\ i \ell\end{array}\right)$, then by Lemma 12, we can throw away an element of $\mathcal{S}_{i}^{*}$ in such a way that $\mathcal{S}_{i}^{*}$ remains $(k-i) \ell$ representative for $\mathcal{S}_{i}$. Therefore, it can be assumed that $\left|\mathcal{S}_{i}^{*}\right| \leq\left(\begin{array}{c}k \ell \\ i \ell\end{array}\right)$. To obtain $\mathcal{S}_{i+1}^{*}$, we enumerate every set $S$ in $\mathcal{S}_{i}^{*}$ and every block $B$, and if $S$ and $B$ are disjoint and $S \cup B$ is independent, then $S \cup B$ is put into $\mathcal{S}_{i+1}^{*}$. We claim that the resulting system is $(k-i-1) \ell$-representative for $\mathcal{S}_{i+1}$ provided that $\mathcal{S}_{i}^{*}$ is $(k-i) \ell$-representative for $\mathcal{S}_{i}$. Assume that there is a set $X \in \mathcal{S}_{i+1}$ and a set $Y$ of size $(k-i-1) \ell$ such that $X \cap Y=\emptyset$ and $X \cup Y$ is independent. By definition, $X$ is the union of $i+1$ blocks; let $B$ be an arbitrary block of $X$. Let $X_{0}=X \backslash B$ and $Y_{0}=Y \cup B$. Now $X_{0}$ is in $\mathcal{S}_{i}$, and we have $X_{0} \cap Y_{0}=\emptyset$ and $X_{0} \cup Y_{0}=X \cup Y$ is independent. Therefore, there is a set $X_{0}^{*} \in \mathcal{S}_{i}^{*}$ with $X_{0}^{*} \cap Y_{0}=\emptyset$ and $X_{0}^{*} \cup Y_{0}$ independent. This means that the independent set $X^{*}:=X_{0}^{*} \cup B$ is put into $\mathcal{S}_{i+1}^{*}$, and it satisfies $X^{*} \cap Y=\emptyset$ and $X^{*} \cup Y$ independent.

When constructing the set system $\mathcal{S}_{i+1}^{*}$, the amount of work to be done is polynomial in $\left\|A^{\prime}\right\|$ for each member $S$ of $\mathcal{S}_{i}^{*}$. As discussed above, the size of each $\mathcal{S}_{i}^{*}$ can be bounded by $\left(\begin{array}{c}k \ell \\ i \ell\end{array}\right)$, thus the running time is $f(k, \ell) \cdot\left\|A^{\prime}\right\|^{O(1)}$. 


\section{Applications}

In this section we derive some consequences of the main result: we list problems that can be solved using the algorithm of Theorem 1 .

\subsection{Matroid Intersection}

Given matroids $M_{1}\left(E, \mathcal{I}_{1}\right), \ldots, M_{\ell}\left(E, \mathcal{I}_{\ell}\right)$ over a common ground set, their intersection is the set system $\mathcal{I}_{1} \cap \cdots \cap \mathcal{I}_{\ell}$. In general, the resulting set system is not a matroid, even for $k=2$. Deciding whether there is a $k$-element set in the intersection of two matroids is polynomial-time solvable (cf. [9]), but NPhard for more than two matroids. Here we show that the problem is randomized fixed-parameter tractable for a fixed number of represented matroids:

Theorem 13. Let $M_{1}, \ldots, M_{\ell}$ be matroids over the same set, given by their representations $A_{1}, \ldots, A_{\ell}$ over $F$. We can decide in $f(k, \ell) \cdot\left(\sum_{i=1}^{\ell}\left\|A_{i}\right\|\right)^{O(1)}$ randomized time if there is a $k$-element set that is independent in every $M_{i}$.

Proof. Let $E=\left\{e_{1}, \ldots, e_{n}\right\}$. We rename the elements of the matroids to make the ground sets pairwise disjoint: let $e_{j}^{(i)}$ be the copy of $e_{j}$ in $M_{i}$. By Prop. 5 , a representation of $M:=M_{1} \oplus \cdots \oplus M_{\ell}$ can be obtained. Partition the ground set of $M$ into blocks of size $\ell$ : for $1 \leq j \leq n$, block $B_{j}$ is $\left\{e_{j}^{(1)}, \ldots, e_{j}^{(\ell)}\right\}$. If $M$ has an independent set that is the union of $k$ blocks, then the corresponding $k$ elements of $E$ is independent in each of $M_{1}, \ldots, M_{\ell}$. Conversely, if $X \subseteq E$ is independent in every matroid, then the union of the corresponding blocks is independent in $M$. Therefore, the algorithm of Theorem 1 answers the question.

\subsection{Disjoint Sets}

Packing problems form a well-studied class of combinatorial optimization problems. Here we study the case when the objects to be packed are small:

Theorem 14. Let $\mathcal{S}=\left\{S_{1}, \ldots, S_{n}\right\}$ be a collection of subsets of $E$, each of size at most $\ell$. There is an $f(k, \ell) \cdot n^{O(1)}$ time randomized algorithm for deciding whether it is possible to select $k$ pairwise disjoint subsets from $\mathcal{S}$.

Proof. By adding dummy elements, it can be assumed that each $S_{i}$ is of size exactly $\ell$. Let $V=\left\{v_{i, j}: 1 \leq i \leq n, 1 \leq j \leq \ell\right\}$. We define a partition matroid over $V$ as follows. For every element $e \in E$, let $V_{e} \subseteq V$ contain $v_{i, j}$ if and only if the $j$-th element of $S_{i}$ is $e$. Clearly, the $V_{e}$ 's form a partition of $V$. Consider the partition matroid $M$ where a set is independent if and only if it contains at most 1 element from each class of the partition. Let block $B_{i}$ be $\left\{v_{i, 1}, \ldots, v_{i, \ell}\right\}$. If $k$ disjoint sets can be selected from $\mathcal{S}$, then the union of the corresponding $k$ blocks is independent in $M$ as every element is contained in at most one of the selected sets. The converse is also true: if the union of $k$ blocks is independent, then the corresponding $k$ sets are disjoint, hence the result follows from Theorem 1 .

Theorem 14 immediately implies the existence of randomized fixed-parameter tractable algorithms for two well-know problems: DisJoInT TRIANGLES and 
Edge Disjoint Triangles. In these problems the task is to find, given a graph $G$ and an integer $k$, a collection of $k$ triangles that are pairwise (edge) disjoint. If $E$ is the set of vertices (edges) of $G$, and the sets in $\mathcal{S}$ are the triangles of $G$, then it is clear that the algorithm of Theorem 14 solves the problem.

\subsection{Feedback Edge Set with Budget Vectors}

Given a graph $G(V, E)$, a feedback edge set is a subset $X$ of edges such that $G(V, E \backslash X)$ is acyclic. If the edges of the graph are weighted, then finding a minimum weight feedback edge set is the same as finding a maximum weight spanning forest, hence it is polynomial time solvable. Here we study a generalization of the problem, where each edge has a vector of integer weights:

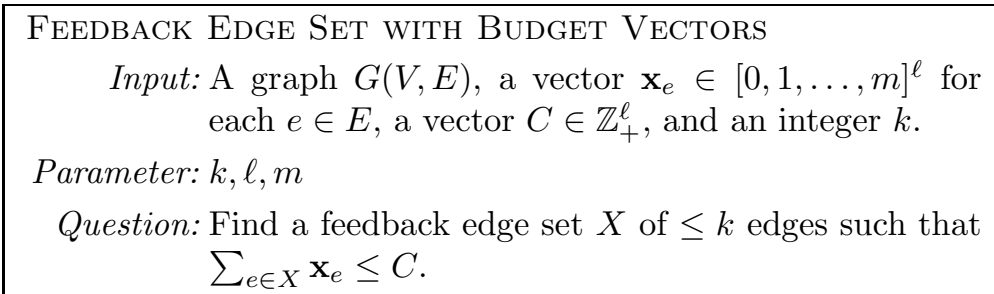

Theorem 15. Feedback Edge Set with Budget Vectors can be solved in $f(k, \ell, m) \cdot n^{O(1)}$ randomized time.

Proof. It can be assumed that $k=|E|-|V|+c(G)$ (where $c(G)$ is the number of components of $G$ ): if $k$ is smaller, then there is no solution; if $k$ is larger, then it can be decreased without changing the problem. Let $M_{0}\left(E, \mathcal{I}_{0}\right)$ be the dual of the cycle matroid of $G$. The rank of $M_{0}$ is $k$, and a set $X$ of $k$ edges is a basis of $M$ if and only if the complement of $X$ is a spanning forest.

Let $C=\left[c_{1}, \ldots, c_{\ell}\right]$ and $n=|E|$. For $i=1, \ldots, \ell$, let $M_{i}\left(E_{i}, \mathcal{I}_{i}\right)$ be the uniform matroid $U_{n m, c_{i}}$. By Props. 9, 4, 7, 6, and 5, a representation of the direct sum $M=M_{0} \oplus M_{1} \oplus \cdots \oplus M_{k}$ can be constructed in polynomial time. For each $e \in E$, let $B_{e}$ be a block containing $e \in E$ and $x_{e}^{(i)}$ arbitrary elements of $E_{i}$ for every $i=1, \ldots, \ell$ (where $x_{e}^{(i)} \leq m$ denotes the $i$-th component of $\mathbf{x}_{e}$ ). The set $E_{i}$ contains $n m$ elements, which is sufficiently large to make the blocks $B_{i}$ disjoint. The size of each block is at most $\ell^{\prime}:=1+m \ell$, hence the algorithm of Theorem 1 can be used to determine in $f\left(k, \ell^{\prime}\right) \cdot n^{O(1)}$ randomized time whether there is an independent set that is the union of $k$ blocks. It is clear that every such independent set corresponds to a feedback edge set such that the total weight of the edges does not exceed $C$ at any component.

\subsection{Reliable Terminals}

In this section we give a randomized fixed-parameter tractable algorithm for a combinatorial problem motivated by network design applications. 


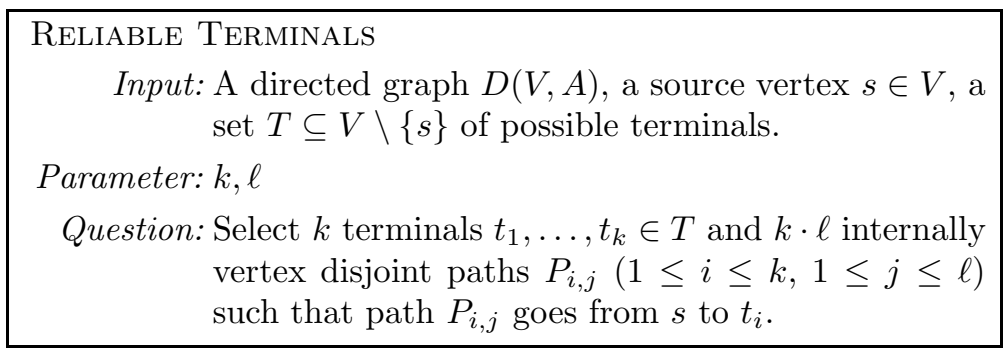

The problem models the situation when $k$ terminals have to be selected that receive $k$ different data streams (hence the paths going to different terminals should be disjoint due to capacity constraints) and each data stream is protected from $\ell-1$ node failures (hence the $\ell$ paths of each data stream should be disjoint).

Let $D(V, A)$ be a directed graph, and let $S \subseteq A$ be a subset of vertices. We say that a subset $X \subseteq S$ is linked to $S$ if there are $|X|$ vertex disjoint paths going from $S$ to $X$. (Note that here we require that the paths are disjoint, not only internally disjoint. Furthermore, zero-length paths are also allowed if $X \cap S \neq \emptyset$.) A result due to Perfect shows that the set of linked vertices form a matroid:

Theorem 16 (Perfect [7]). Let $D(V, A)$ be a directed graph, and let $S \subseteq A$ be a subset of vertices. The subsets that are linked to $S$ form the independent sets of a matroid over $V$. Furthermore, a representation of this matroid can be obtained in randomized polynomial time.

Proof. Let $V=\left\{v_{1}, \ldots, v_{n}\right\}$ and assume that no arc enters $S$. Let $G(U, W ; E)$ be a bipartite graph where a vertex $u_{i} \in U$ corresponds to each vertex $v_{i} \in V$, and a vertex $w_{i} \in W$ corresponds to each vertex $v_{i} \in V \backslash S$. For each $v_{i} \in V$, there is an edge $w_{i} u_{i} \in E$, and for each $\overrightarrow{v_{i} v_{j}} \in A$, there is an edge $u_{i} w_{j} \in E$.

The size of a maximum matching in $G$ is at most $|W|=n-|S|$. Furthermore, a matching of size $n-|S|$ can be obtained by taking the edges $u_{i} w_{i}$ for every $v_{i} \notin S$. Let $V_{0} \subseteq V$ be a subset of size $|S|$, and let $U_{0}$ be the corresponding subset of $U$. We claim that $V_{0}$ is linked to $S$ if and only $G$ has a matching covering $U \backslash U_{0}$. Assume first that there are $|S|$ disjoint paths going from $S$ to $V_{0}$. Consider the matching where $w_{i} \in W$ is matched to $u_{j}$ if one of the paths enters $v_{i}$ from $v_{j}$, and $w_{i}$ is matched to $u_{i}$ otherwise. This means that $u_{i}$ is matched if one of the paths reaches $v_{i}$ and continues further on, or if none of the paths reaches $v_{i}$. Thus the unmatched $u_{i}$ 's corresponds to the end points of the paths, as required.

To see the other direction, consider a matching covering $U \backslash U_{0}$. As $\left|U \backslash U_{0}\right|=$ $n-|S|$, this is only possible if the matching fully covers $W$. Let $v_{i_{1}}$ be a vertex of $S$. Let $u_{i_{2}}$ be the pair of $w_{i_{1}}$ in the matching, let $u_{i_{3}}$ be the pair of $w_{i_{2}}$, etc. We can continue this until a vertex $u_{i_{k}}$ is found that is not covered in the matching. Now $v_{i_{1}}, v_{i_{2}}, \ldots, v_{i_{k}}$ is a path going from $S$ to $v_{i_{k}} \in V_{0}$. If this procedure is repeated for every vertex of $S$, then we obtain $|S|$ paths that are pairwise disjoint, and each of them ends in a vertex of $V_{0}$.

If $X$ is linked to $S$, then $X$ can be extended to a linked set of size exactly $|S|$ by adding vertices of $S$ to it (as they are connected to $S$ by zero-length paths). The observation above shows that linked sets of size $|S|$ are exactly the bases of 
the dual of the transversal matroid of $G$, which means that the linked sets are exactly the independent sets of this matroid. By Props. 10 and 7, a representation of this matroid can be constructed in randomized polynomial time.

Theorem 17. Reliable TeRminals is solvable in $f(k, \ell) \cdot n^{O(1)}$ randomized time.

Proof. Let us replace the vertex $s$ with $k \cdot \ell$ independent vertices $S=\left\{s_{1}, \ldots, s_{k \ell}\right\}$ such that each new vertex has the same neighborhood as $s$. Similarly, each $t \in T$ is replaced with $\ell$ vertices $t^{(1)}, \ldots, t^{(\ell)}$, but now we remove every outgoing edge from $t^{(2)}, \ldots, t^{(\ell)}$. Denote by $D^{\prime}$ the new graph. It is easy to see that a set of terminals $t_{1}, \ldots, t_{k}$ form a solution for the RELIABLE TERMinals problem if and only if the set $\left\{t_{i}^{(j)}: 1 \leq i \leq k, 1 \leq j \leq \ell\right\}$ is linked to $S$. Using Theorem 16, we can construct a representation of the matroid whose independent sets are exactly the sets linked to $S$ in $D^{\prime}$. Delete the columns that do not correspond to vertices in $T$, hence the ground set of the matroid has $\ell|T|$ elements. Partition the ground set into blocks of size $\ell$ : for every $t \in T$, there is a block $B_{t}=\left\{t^{1}, \ldots, t^{\ell}\right\}$. Clearly, the Reliable Terminals problem has a solution if and only if the matroid has an independent set that is the union of $k$ blocks. Therefore, Theorem 1 can be used to solve the problem.

\section{References}

1. R. G. Downey and M. R. Fellows. Parameterized complexity. Monographs in Computer Science. Springer-Verlag, New York, 1999.

2. R. L. Graham, M. Grötschel, and L. Lovász, editors. Handbook of combinatorics. Vol. 1, 2. Elsevier Science B.V., Amsterdam, 1995.

3. L. Lovász. Flats in matroids and geometric graphs. In Combinatorial surveys (Proc. Sixth British Combinatorial Conf., Royal Holloway Coll., Egham, 1977), pages 45-86. Academic Press, London, 1977.

4. L. Lovász. Matroid matching and some applications. J. Combin. Theory Ser. B, 28(2):208-236, 1980.

5. D. Marx. Parameterized coloring problems on chordal graphs. Theoret. Comput. Sci., 351(3):407-424, 2006.

6. B. Monien. How to find long paths efficiently. In Analysis and design of algorithms for combinatorial problems (Udine, 1982), volume 109 of North-Holland Math. Stud., pages 239-254. North-Holland, Amsterdam, 1985.

7. H. Perfect. Applications of Menger's graph theorem. J. Math. Anal. Appl., 22:96$111,1968$.

8. J. Plehn and B. Voigt. Finding minimally weighted subgraphs. In Graph-theoretic concepts in computer science (WG'90), LNCS 484, 18-29. Springer, Berlin, 1991.

9. A. Recski. Matroid theory and its applications in electric network theory and statics, Springer-Verlag, Berlin, New York and Akadémiai Kiadó, Budapest, 1989.

10. J. T. Schwartz. Fast probabilistic algorithms for verification of polynomial identities. J. Assoc. Comput. Mach., 27(4):701-717, 1980.

11. V. Shoup. Fast construction of irreducible polynomials over finite fields. J. Symbolic Comput., 17(5):371-391, 1994.

12. R. Zippel. Probabilistic algorithms for sparse polynomials. In Symbolic and algebraic computation (EUROSAM 'r9), LNCS 72, 216-226. Springer, Berlin, 1979. 